\title{
Diseño y fiabilidad de un cuestionario sobre la comprensión auditiva/audiovisual
}

\author{
Juan Carlos Casañ Núñez \\ Universitat Politècnica de València, España
}

Artículo recibido 3/11/16, aceptado 25/02/17, versión final 29/04/17

DOI: https://doi.org/10.5565/rev/jtl3.686

\begin{abstract}
Resumen
Este trabajo forma parte de una investigación mixta multifase que explora el empleo de preguntas de comprensión audiovisual integradas en el vídeo en forma de subtítulos y sincronizadas con los fragmentos pertinentes para el aprendizaje de lenguas extranjeras. El objetivo principal del artículo es comunicar el diseño y la fiabilidad test-retest de un cuestionario sobre la comprensión auditiva/audiovisual para estudiantes adultos de español como lengua extranjera que sería administrado en estudios posteriores. Además, se analizan las respuestas de los informantes para conocer algunas de sus creencias y preferencias. Veinte aprendientes completaron el cuestionario en dos ocasiones con un intervalo de catorce días. Los resultados indican que el instrumento es fiable. Asimismo, la mayoría de los participantes considera que es importante practicar la comprensión auditiva/audiovisual en el aula y que la imagen del vídeo ayuda a comprender a los interlocutores. Finalmente se discuten las aplicaciones y limitaciones del estudio.
\end{abstract}

Palabras clave: comprensión auditiva, comprensión audiovisual, cuestionario, fiabilidad test-retest, subtitulación

\begin{abstract}
The study reported in this paper is part of a multiphase mixed-methods investigation that explores the usage of audiovisual comprehension questions embedded within a video sequence in the form of subtitles and synchronized with the relevant fragments, for the purpose of language learning. The main objective of this paper is to account for the design and test-retest reliability of a questionnaire on listening/viewing comprehension for adult learners of Spanish as a foreign language that would be administered in subsequent studies. In addition, informants' answers are analysed in order to extract information about their beliefs and preferences. Twenty learners completed the questionnaire twice, fourteen days apart. The results show that the instrument is reliable. Furthermore, most of the participants consider that it is important to practise listening/viewing comprehension in the language class and that video images aid in understanding speakers. To conclude, the applications and limitations of the study are discussed.
\end{abstract}

Key words: listening, viewing comprehension, questionnaire, test-retest reliability, subtitling

\section{Resum}

Aquest treball forma part d'una investigació mixta multifase que explora la utilització de preguntes de comprensió audiovisual integrades al vídeo en forma de subtítols i sincronitzades amb els fragments pertinents per a l'aprenentatge de llengües estrangeres. L'objectiu principal de l'article és 
comunicar el disseny i la fiabilitat test-retest d'un qüestionari sobre la comprensió auditiva/audiovisual per a estudiants adults d'espanyol com a llengua estrangera que seria administrat en estudis posteriors. A més, s'analitzen les respostes dels informants per conèixer algunes de les seves creences i preferències. Vint aprenents van completar el qüestionari en dues ocasions amb un interval de catorze dies. Els resultats indiquen que l'instrument és fiable. Així mateix, la majoria dels participants considera que és important practicar la comprensió auditiva/audiovisual a l'aula i que la imatge del vídeo ajuda a comprendre als interlocutors. Finalment es discuteixen les aplicacions i limitacions de l'estudi.

Paraules clau: comprensió auditiva, comprensió audiovisual, qüestionari, fiabilitat test-retest, subtitulació

\section{Introducción}

Las grabaciones en vídeo deberían predominar sobre las grabaciones de audio para practicar la comprensión auditiva/audiovisual en el aula de lenguas extranjeras (Lynch, 2009; Mendelsohn, 1994; Ur, 1999). En primer lugar, es coherente con una concepción de esta actividad comunicativa como proceso de interpretación de información auditiva y visual, como defienden autores como Harris (2003), Lynch (2012), Riley (1979) y Rubin (1995). En segundo lugar, el vídeo permite observar la realidad de la mayor parte de nuestras interacciones orales: conversamos con personas (y no solo voces) y en un contexto determinado. En tercer lugar, el vídeo tiene un efecto favorable en la motivación (Flowerdew \& Miller, 2005; Ur, 1999; Vandergrift \& Goh, 2012). Además, hay argumentos a favor de un aprendizaje multimodal (v. Mayer, 2009). Según Plass y Jones (2005, p. 479) "it is the combination of both visual and verbal presentations of information that has most strongly and consistently supported listening and reading comprehension and vocabulary acquisition".

Los especialistas sugieren la explotación de los textos orales en tres fases: antes, durante y después de la audición/visionado (v. Flowerdew \& Miller, 2005; Giovannini, Martín Peris, Rodríguez \& Simón, 1996; Richards \& Burns, 2012; Rost, 2016). Con respecto a las actividades en la fase de audición, Underwood (1989) enfatiza la necesidad de que sean sencillas por la complejidad de escuchar, leer y escribir simultáneamente. Las tareas con vídeo pueden resultar todavía más complicadas. Por un lado, existe un conflicto de atención visual entre observar un vídeo y completar una actividad escrita. Este conflicto contribuye a explicar el relativamente bajo grado de atención a la imagen en investigaciones que han estudiado el comportamiento visual de los aprendientes mientras realizan tareas de comprensión simultáneas a la emisión del vídeo. Aunque Ockey (2007) 
no indica la media, a partir del tiempo que cada informante mira hacia la pantalla se puede calcular que es el $44.9 \%$ del tiempo. Wagner (2007) obtiene un promedio del $69 \%$, Wagner (2010) $47.9 \%$ y Suvorov (2015) $50.7 \%$ en context videos y $58 \%$ en content videos. Por otro lado, la capacidad de la memoria de trabajo para procesar información nueva es muy limitada (Sweller, Ayres \& Kalyuga, 2011) y, por ello, escuchar, ver, leer y escribir al mismo tiempo llegar a saturarla.

Este artículo es parte de una investigación mixta multifase (Creswell, 2014; Creswell \& Plano Clark, 2011) que explora la utilización de preguntas de comprensión audiovisual integradas en el vídeo en forma de subtítulos y sincronizadas con los fragmentos relevantes para el aprendizaje y evaluación de lenguas extranjeras (v. Casañ Núñez, 2015a, 2015b, 2016, 2017). Esta técnica podría aportar varios beneficios frente a visionados con los materiales de la actividad solo en papel. Entre ellos, podría reducir el conflicto de atención visual, al aproximar espacial y temporalmente las preguntas y los fragmentos pertinentes; y podría minimizar la carga cognitiva de la actividad, al posibilitar que el aprendiente solo tenga que atender a una pregunta subtitulada en cada momento y no a varias cuestiones en papel simultáneamente. La estrategia está destinada a estudiantes adultos de lenguas extranjeras. Se considera especialmente adecuada para aprendientes de niveles iniciales porque, según Field (2008), son los que pueden ver sobrecargada su memoria de trabajo más fácilmente. Además, se contempla su utilización puntual en otros niveles por dos motivos. En primer lugar, favorece que se mantenga el plano de trabajo en pantalla frente a visionados con las preguntas solo en papel. En segundo lugar, las opiniones sobre esta técnica de estudiantes con un nivel de comprensión audiovisual en español como lengua extranjera (ELE) $\mathrm{B} 2^{+} / \mathrm{C} 1$ son globalmente positivas (Casañ Núñez, 2016).

El trabajo actual tenía el objetivo fundamental de comunicar el diseño y la fiabilidad de un cuestionario sobre la comprensión auditiva/audiovisual para estudiantes de ELE que se administraría en estudios posteriores. Además, se pretendía responder a las siguientes preguntas de investigación:

(1) ¿Qué importancia atribuían los estudiantes a la práctica de la comprensión auditiva/audiovisual en clase para aprender español?

(2) Sobre la base de sus experiencias como estudiantes de español, ¿qué tipo de grabaciones (audio o vídeo) preferían para practicar la comprensión auditiva/audiovisual en el aula? 
(3) Según sus experiencias aprendiendo lenguas extranjeras, ¿consideraban que la imagen del vídeo los ayudaba a comprender lo que decían los interlocutores?

(4) ¿Cómo practicaban la comprensión auditiva/audiovisual fuera del aula?

El resto del artículo se estructura del modo siguiente. En primer lugar, se describe la elaboración del instrumento desde su concepción hasta llegar a la versión que sería sometida a un análisis de fiabilidad. En segundo lugar, se refiere el estudio efectuado para determinar la estabilidad y se responde a las preguntas de investigación.

\section{Diseño del cuestionario}

El diseño del cuestionario consta de dos etapas interrelacionadas: planificación y construcción/experimentación. En la fase de planificación se describen los siguientes aspectos: propósito, destinatarios, necesidad, extensión, lengua del cuestionario y de la respuesta. La fase de construcción/experimentación implicó varias etapas. La primera versión se redactó a partir de las necesidades de investigación, las recomendaciones metodológicas de varios autores sobre el diseño de cuestionarios y la consulta de instrumentos sobre el mismo tema. Posteriormente, fue revisado por expertos y se reformuló. A continuación, se identificaron y tradujeron a la lengua materna del grupo meta los posibles términos problemáticos. Por último, se efectuó una prueba piloto y se escribió la versión final.

\section{Planificación}

El propósito del instrumento es recabar ciertas informaciones de los estudiantes de ELE que participen en estudios cuasiexperimentales sobre el uso de preguntas de comprensión audiovisual sobreimpresas. El cuestionario pretende recoger datos sociodemográficos, conocer qué importancia atribuyen a la práctica de la comprensión auditiva/audiovisual en clase para aprender español, averiguar algunas preferencias de aprendizaje sobre la comprensión auditiva/audiovisual, saber si consideran que la imagen del vídeo los ayuda a comprender a los interlocutores, y conocer cómo practican la comprensión auditiva/audiovisual fuera del aula. Estas informaciones son relevantes porque permiten caracterizar a la muestra y trazar relaciones con otros datos obtenidos en esos estudios cuasiexperimentales.

Los destinatarios son estudiantes adultos de ELE capaces de ver cine subtitulado. Se prevé hacer adaptaciones menores del instrumento en función de la muestra. Estas modificaciones pueden ser de dos índoles. Por un lado, cuando se considere que algún 
término en español pueda revestir alguna dificultad para los participantes, se incorporarán traducciones entre paréntesis, bien a la lengua materna de los informantes en caso de grupos monolingües, bien a la lengua habitual de comunicación en caso de muestras no monolingües. Por otro lado, puede ser conveniente hacer alteraciones en la sección de aspectos sociodemográficos. En este trabajo se describe la versión para estudiantes de la asignatura de ELE de nivel A2 ${ }^{+}$Español II impartida en la Facultad de Letras de la Universidade de Coimbra (FLUC).

Se optó por elaborar un cuestionario porque "they are relatively easy to construct, extremely versatile and uniquely capable of gathering a large amount of information quickly in a form that is readily processible" (Dörnyei, 2007, p. 101). Es necesario diseñarlo porque se desconoce la existencia de un cuestionario en español que trate todos los aspectos mencionados y que esté destinado a la misma población. Debe ser conciso para facilitar su administración junto con los otros instrumentos previstos en los estudios cuasiexperimentales: un test de comprensión audiovisual y un cuestionario postest. En cuanto al idioma de la redacción, se utiliza la lengua meta porque no todos los informantes comparten idioma materno. En relación con la lengua de respuesta, se permite utilizar tanto el español como la lengua materna o la habitual en la comunicación (en este caso, el portugués) para que los participantes puedan expresarse sin dificultades.

\section{Construcción}

El primer borrador se elaboró a partir de la consideración de las necesidades de investigación y de las recomendaciones metodológicas de varios especialistas (Brown, 2001; Cohen, Manion, \& Morrison, 2011; Creswell, 2012; De Vaus, 2002; Dörnyei, 2003, 2007; Murillo Torrecilla, 2004), y de la consulta de cuestionarios que abordaban contenidos relacionados (Coniam, 2001; García-Romeu, 2006; Minera Reyna, 2010; Sueyoshi \& Hardison, 2005; Suvorov, 2008). Esta primera versión fue revisada por dos expertas en lingüística aplicada a la enseñanza de lenguas, la Dra. Ana Gimeno (Universitat Politècnica de València) y la Dra. Virginia González (Universitat de València), y posteriormente modificada. El siguiente paso consistió en identificar los posibles términos problemáticos en español para los destinatarios (los estudiantes de Español II de la FLUC) y en situar junto a ellos una traducción a una lengua que todos conocieran. Como la inmensa mayoría de los informantes eran lusófonos, y los que no lo eran tenían conocimientos de portugués, el idioma de la traducción fue la lengua de Camões. La identificación de las palabras y la elección de las traducciones fueron posibles 
gracias a la propia experiencia docente con estudiantes de Español II en ediciones anteriores, y a las sugerencias de una profesora de portugués con conocimientos de español, la Dra. Cláudia Cravo (FLUC). Semanas después se efectuó una prueba piloto con diez estudiantes que acababan de cursar Español I, la asignatura que precede a Español II en la FLUC. El objetivo era experimentar todo el proceso de recogida de datos para averiguar si las indicaciones proporcionadas durante la administración y la redacción de los ítems eran claras. Asimismo, se pretendía detectar problemas no anticipados. Se consideró que las instrucciones y la formulación de los ítems eran comprensibles porque ningún informante manifestó tener dificultades, y porque no se encontraron respuestas en blanco ni incongruentes en sí mismas o en relación con otras. Algunos participantes indicaron categorías de respuesta no contempladas. Estas alternativas fueron incorporadas en la versión siguiente, y a la postre definitiva, del instrumento.

\section{Estructura}

El cuestionario presenta cinco partes: título, nombre, texto introductorio, ítems y un apartado en el que se puede añadir información adicional (v. anexo). El título pretende ser informativo y activar el conocimiento previo de los participantes. Se pide a los informantes que indiquen su nombre para poder trazar relaciones con los otros instrumentos del estudio cuasiexperimental, y si fuera pertinente, con otras actividades o pruebas que se efectúen durante el semestre en la asignatura Español II. El tercer apartado lo conforma un texto introductorio en el que se especifica el objetivo del cuestionario, se menciona que la información recogida se tratará de forma confidencial y que solo se empleará con fines de investigación, y se señala que no hay respuestas correctas o incorrectas porque las contestaciones se basan en las experiencias particulares aprendiendo español. Los márgenes, el interlineado y la separación entre párrafos del instrumento están ajustados para intentar que visualmente parezca lo más breve posible, sin que eso suponga la división de ninguna pregunta en dos páginas.

Los veintiséis ítems del cuestionario se dividen en cuatro áreas: preguntas generales (ítems 1-9), preferencias de aprendizaje (ítems 10-19), valor de la imagen (ítem 20) y aprendizaje fuera del aula (ítems 21-26). Se opta por una división por temáticas porque "saltar de un tema a otro supone un gran esfuerzo al encuestado" (Murillo Torrecilla, 2004, p. 6). La primera sección tiene el propósito de conocer varios datos sociodemográficos de los informantes: la edad (1), los estudios universitarios (2-4), la lengua materna (5), si han cursado Español I en la FLUC (6), cuánto tiempo llevan estudiando español (7), y si han 
estado en países hispanohablantes (8) y, en caso afirmativo, durante cuánto tiempo (9). El bloque "preferencias de aprendizaje" tiene el objetivo de averiguar qué importancia atribuyen a la práctica de la comprensión auditiva/audiovisual en clase (10), saber si el tema del vídeo influye en su motivación (11), conocer qué temas prefieren (12-18), y si tienen alguna preferencia por las grabaciones de audio o vídeo (19). El apartado "valor de la imagen" está constituido por un ítem que pretende averiguar si los participantes consideran que la imagen les ayuda a comprender a los interlocutores (20). En la sección "aprendizaje fuera del aula" se pide a los informantes que indiquen si practican la comprensión auditiva/audiovisual fuera del aula, de qué forma, y con qué frecuencia (2126). La última sección tiene el propósito de permitir que los participantes puedan hacer comentarios adicionales relacionados con el aprendizaje de la comprensión auditiva/audiovisual.

Los ítems se describen según la clasificación de Saris y Gallhofer (2014). Se utilizan preguntas de respuesta abierta $(4,7,9)$ y categórica $(1-3,5-6,8,10-26)$. Se recurre a ítems de respuesta abierta para conocer los posibles estudios previos (4), y para averiguar el tiempo aprendiendo español (7) y pasado en países hispanohablantes (9) porque resulta complicado anticipar las posibles respuestas. Para formular los ítems de respuesta categórica se siguen las recomendaciones de Saris y Gallhofer (2014, pp. 101-103): las opciones de respuesta son mutuamente excluyentes, intentan ofrecer un abanico de opciones lo suficientemente amplio como para abarcar las posibles respuestas de los informantes, están relacionadas con el mismo concepto y "match with the information provided in the request or statement asked". Se emplean dos tipos de ítems categóricos: de respuesta nominal $(2,3,5,6,8$ y 19) y ordinal $(1,10-18,20-26)$.

\section{Fiabilidad}

El estudio descrito en esta sección pretendía determinar la fiabilidad del cuestionario. Además, se quería conocer las creencias y preferencias de los estudiantes de ELE sobre una serie de aspectos relacionados con la comprensión auditiva/audiovisual.

\section{Método}

Se utilizó el método del cuestionario repetido (Brown, 2001, p. 171-172). Esta técnica consiste en administrar el mismo instrumento a las mismas personas con un intervalo de tiempo y en comparar los resultados. Siguiendo las recomendaciones de Brown (2001), Cooper y Schindler (2008) y De Vaus (2002) se eligió un intervalo de dos semanas. Se 
empleó esta técnica porque es habitual en el desarrollo de cuestionarios (Cohen, Manion \& Morrison, 2011) y porque es la única capaz de estimar la fiabilidad de preguntas individuales (De Vaus, 2002). Esta metodología fue empleada por Bere y Bjørkelund (2009), Chang (2005), Hammond et al. (2015) y Nowrouzi, Tam, Nimehchisalem y Zareian (2014) para medir la fiabilidad de cuestionarios en distintas áreas del conocimiento. En la segunda administración se decidió prescindir de los ítems relacionados con datos sociodemográficos por su simplicidad.

\section{Participantes}

La muestra estaba constituida por veinte personas. Todas eran estudiantes de Español II, una asignatura de ELE de nivel $\mathrm{A}^{+}{ }^{+}$ofrecida por la FLUC. Los informantes eran lusófonos y tenían menos de treinta y cinco años. El $75 \%$ era de sexo femenino y el $25 \%$ de sexo masculino. Todos cursaban estudios de primer ciclo: Turismo (35\%), Periodismo (25\%), Estudios Europeos (25\%) y Estudios Artísticos (25\%). Dado que el cuestionario estaba redactado en español, cabe hacer mención al nivel de comprensión lectora en ELE aproximado de los participantes. Por los textos escritos empleados en clase durante el curso (entre otros, una selección de historias de Elvira Lindo y de cuentos de García Márquez), el nivel de comprensión lectora se estimaba en torno al $\mathrm{B} 1^{+}$. Esa discordancia entre el nivel general de la asignatura y el nivel de comprensión escrita lo explica la proximidad entre el español y el portugués. De acuerdo con Trindade Natel (2002: p. 831), el conocimiento previo del estudiante lusófono "funciona como un medio facilitador de la comprensión" en español. Los informantes eran muy similares a la muestra objetivo porque el cuestionario estaba dirigido a los estudiantes de la misma disciplina en su siguiente edición.

\section{Procedimiento}

El autor administró el cuestionario dos veces con un intervalo de dos semanas en clase de Español II en marzo de 2013. En ambas administraciones se destacó la importancia de las respuestas para la investigación, se acentuó que no había respuestas correctas ni incorrectas, y se explicó como marcar las respuestas. También se informó a los participantes de que disponían de todo el tiempo que necesitaran para completar el cuestionario y de que podían plantear cualquier duda que surgiera en español o portugués. Para limitar la posibilidad de que la segunda administración provocase algún rechazo, esta se justificó por las necesidades de la investigación y se señaló que se revelaría el motivo después de la recogida de datos. Mientras respondían, el investigador permaneció en 
silencio y se mantuvo a disposición de los participantes. Ningún informante solicitó ayuda durante la recogida de datos.

\section{Análisis de datos}

Los datos cuantitativos se introdujeron en la versión 21 de SPSS para Windows. Unos días después se verificó que los datos insertados eran correctos. Se hizo igualmente un análisis de frecuencias para comprobar que no había valores anómalos ni perdidos. Se calcularon los coeficientes de correlación de Spearman y las medianas para los datos ordinales (ítems 10-18, 20-26), y el índice de kappa de Cohen y la moda para la pregunta de respuesta nominal (ítem 19). El coeficiente de correlación de Spearman se interpretó de acuerdo con las definiciones de Cohen (1988): .1 = tamaño de efecto pequeño, .3 = tamaño de efecto mediano, у . 5 = tamaño de efecto grande. Esta propuesta es ampliamente citada en la bibliografía (por ejemplo, Field, 2009; Larson-Hall, 2010; Pallant, 2011). El índice de kappa de Cohen se interpretó según la propuesta de Altman (1991: p. 404): los valores de kappa inferiores a .20 indican acuerdo "poor", y los comprendidos entre $.21 \mathrm{y} .40, .41 \mathrm{y}$ $.60, .61$ y .80, y .81 y 1, acuerdo "fair", "moderate", "good" y "very good", respectivamente. Esta escala es utilizada por muchos autores, por ejemplo, por Garmy, Jakobsson y Nyberg (2012) y por Masson et al. (2002). Para la representación de los datos se eligieron tablas porque, de acuerdo con Dörnyei (2007), pueden aportar una descripción más precisa y rica que las figuras.

\section{Resultados y discusión}

Se puede deducir que los participantes no tuvieron dificultades para completar el cuestionario porque nadie manifestó tener dudas y porque no se hallaron valores perdidos ni anómalos en los análisis de frecuencias de los ítems.

El primer objetivo era estimar la fiabilidad test-retest del instrumento. Los resultados sugerían que la estabilidad era buena (v. tablas 1 y 2 ). En los ítems ordinales (10-18, 20-26), se observó una correspondencia casi total en las medianas en las dos administraciones. Asimismo, los coeficientes de correlación de Spearman $\left(r_{s}=.522-.879, p\right.$ $<.05)$ indicaban correlaciones fuertes de acuerdo con la propuesta de Cohen (1998). En cuanto al ítem nominal (19), la moda era la misma en las dos administraciones, y el índice de kappa de Cohen $(k=.747, p<.001)$ mostraba que existía un buen grado de acuerdo según la escala de Altman (1991).

Tabla 1. Datos ordinales: mediana y coeficiente de correlación de Spearman

\begin{tabular}{llllll}
\hline Ítem & $\mathbf{n}$ & $\begin{array}{l}\text { Mediana } \\
\text { test }\end{array}$ & $\begin{array}{l}\text { Mediana } \\
\text { retest }\end{array}$ & $\begin{array}{l}\text { Rho } \\
\text { Spearman }\end{array}$ & $\begin{array}{l}\text { Sig. } \\
\text { (bilateral) }\end{array}$ \\
\hline
\end{tabular}




\begin{tabular}{|c|c|c|c|c|c|}
\hline $\begin{array}{l}\text { 10. Importancia de la comprensión } \\
\text { auditiva/audiovisual }\end{array}$ & 20 & 4 & 4.5 & $.622 * *$ & .003 \\
\hline 11. Influencia del tema en la motivación & 20 & 4 & 4 & $.597 * *$ & .005 \\
\hline 12. Interés por el cine & 20 & 4 & 4 & $.739 * *$ & .000 \\
\hline 13. Interés por la cocina & 20 & 3 & 3 & $.584 * *$ & .007 \\
\hline 14. Interés por el deporte & 20 & 3 & 3 & $.579 * *$ & .007 \\
\hline 15. Interés por la historia & 20 & 4 & 4 & $.845 * *$ & .000 \\
\hline 16. Interés por la música & 20 & 4 & 4 & $.806^{* *}$ & .000 \\
\hline 17. Interés por las noticias de actualidad & 20 & 4 & 4 & $.522 *$ & .018 \\
\hline 18. Interés por las fiestas tradicionales & 20 & 4 & 4 & $.748 * *$ & .000 \\
\hline 20. Grado de ayuda de la imagen & 20 & 4 & 4 & $.532 *$ & .016 \\
\hline 21. Escuchar grabaciones de audio para ELE & 20 & 2 & 1.5 & $.879 * *$ & .000 \\
\hline $\begin{array}{l}\text { 22. Escuchar grabaciones de audio para } \\
\text { nativos }\end{array}$ & 20 & 2 & 2 & $.621 * *$ & .003 \\
\hline 23. Hablar con hispanohablantes & 20 & 2 & 2 & $.709^{* *}$ & .000 \\
\hline $\begin{array}{l}\text { 24. Hablar con no hispanohablantes en } \\
\text { español }\end{array}$ & 20 & 2 & 2 & $.654 * *$ & .002 \\
\hline 25. Ver grabaciones de vídeo para ELE & 20 & 1 & 1 & $.541 *$ & .014 \\
\hline 26. Ver grabaciones de vídeo para nativos & 20 & 2 & 2 & $.560 *$ & .010 \\
\hline
\end{tabular}

*. La correlación es significativa al nivel .05

**. La correlación es significativa al nivel .01

Tabla 2. Datos nominales: moda y kappa de Cohen

\begin{tabular}{llllll}
\hline Ítem & n & Moda test & Moda retest & Kappa de Cohen & Sig. exacta \\
\hline 19. Grabaciones que se prefieren & 20 & 3 & 3 & .747 & .000 \\
\hline
\end{tabular}

El segundo objetivo consistía en averiguar las creencias y preferencias de los aprendices de ELE sobre una serie de aspectos relacionados con la comprensión auditiva/audiovisual. Para investigar estas cuestiones se tuvieron en consideración las respuestas obtenidas en la primera administración del instrumento. ¿Qué importancia atribuían los estudiantes a la práctica de la comprensión auditiva/audiovisual en clase para aprender español? (ítem 10 del cuestionario). Todos los participantes excepto uno consideraban que practicar la comprensión auditiva/audiovisual en el aula era importante (11/55\%) o muy importante (8 / $40 \%$ ). Esos resultados están en sintonía con el peso de la comprensión auditiva/ audiovisual en la comunicación ("we listen to twice as much language as we speak, four times as much as we read, and five times as much as we write", Celce-Murcia \& Olshtain, 2000, p. 102) y en la adquisición ("in order for acquirers to progress to the next stage in the acquisition of the target language, they need to understand input language that includes a structure that is part of the next stage", Krashen \& Terrel, 1983, p. 32).

Sobre la base de sus experiencias como estudiantes de español, ¿qué tipo de grabaciones (audio o vídeo) preferían para practicar la comprensión auditiva/audiovisual en el aula? (ítem 19 del cuestionario). La mayoría de los informantes (11/55\%) no mostró predilección por unos materiales u otros. Asimismo, más participantes preferían las grabaciones en vídeo (6/30 \%) que las de audio (3/15\%). Dado que no se pidió a los 
estudiantes que justificaran la elección, no se puede saber cuáles fueron sus motivaciones. Es posible que la preferencia por el vídeo de algunos informantes esté relacionada con el efecto positivo del vídeo en la motivación (Flowerdew \& Miller, 2005; Ur, 1999; Vandergrift \& Goh, 2012).

Según sus experiencias aprendiendo lenguas extranjeras, ¿consideraban que la imagen del vídeo los ayudaba a comprender lo que decían los interlocutores? (ítem 20 del cuestionario). La mayoría de los participantes respondió que el input visual ayudaba mucho (11 / 55 \%) o muchísimo (4 / 20 \%). Los resultados guardan relación con los obtenidos por Sueyoshi y Hardison (2005) y por Wagner (2010). Sueyoshi y Hardison preguntaron a 42 estudiantes de inglés como segunda lengua si estaban de acuerdo con los siguientes enunciados: "it is easier to understand English when I can see the speaker's face", "it is easier to understand English when I can see the speaker's gestures" and "it is easier to understand English conversations on TV than on the radio" (p. 697). Los participantes mostraron acuerdo con las tres afirmaciones. Para investigar la actitud hacia el uso del vídeo de los estudiantes de inglés como segunda lengua, Wagner (2010) administró un cuestionario a 56 aprendices después de completar un test de comprensión con textos audiovisuales. Las opiniones de los informantes fueron globalmente positivas. En contraste con estos estudios, cabe mencionar investigaciones en las que los estudiantes valoraron negativamente el uso del vídeo en pruebas de comprensión (Coniam, 2001; Suvorov, 2008). Estos resultados aparentemente contradictorios podrían deberse a multitud de elementos: el conflicto de atención visual entre ver el vídeo y completar la tarea, la complejidad de la actividad y del texto para los informantes, la focalización de las preguntas de comprensión audiovisual, el origen de texto (auténtico o creado por los investigadores), la calidad del input, el estrés generado por la prueba, etc.

Por último se quería averiguar ¿cómo practicaban la comprensión auditiva/audiovisual fuera del aula? (ítems 21-26 del cuestionario). Como puede observarse en la tabla 3, la manera más habitual para practicar la comprensión auditiva/audiovisual era ver vídeos para hablantes nativos, seguido por escuchar grabaciones de audio para nativos. La preferencia por el vídeo está en sintonía con la idea defendida por autores como Lynch (2009), Mendelsohn (1994) y Ur (1999) de que los materiales audiovisuales deberían predominar sobre las grabaciones de audio para practicar la comprensión auditiva/audiovisual. Además, la predilección por los materiales auténticos está en consonancia con el valor otorgado al uso de realia en varios enfoques vigentes para 
la enseñanza de lenguas, por ejemplo, el enfoque comunicativo y el enfoque por tareas. En relación con los resultados de "hablar en español con hablantes nativos" y "hablar en español con personas no nativas", parecían lógicos teniendo en cuenta que el español se aprendía como lengua extranjera, por un lado, y que en Coimbra había posibilidades de interactuar con estudiantes de intercambio, residentes y turistas hispanohablantes.

Tabla 3. ¿Cómo practicaban la comprensión auditiva/audiovisual fuera del aula?

\begin{tabular}{|c|c|c|c|c|c|}
\hline & \multicolumn{5}{|c|}{ Porcentaje } \\
\hline & $0^{\mathrm{a}}$ & $1-2$ & $3-4$ & $5-6$ & 7 \\
\hline Escuchar grabaciones de audio para personas que aprenden ELE & $45 \%$ & $45 \%$ & $10 \%$ & & \\
\hline Escuchar grabaciones de audio para nativos & $30 \%$ & $35 \%$ & $35 \%$ & & \\
\hline Hablar en español con hablantes nativos & $40 \%$ & $20 \%$ & $15 \%$ & $25 \%$ & \\
\hline Hablar en español con personas no nativas & $30 \%$ & $35 \%$ & $15 \%$ & $20 \%$ & \\
\hline Ver vídeos para personas que aprenden ELE & $60 \%$ & $20 \%$ & $15 \%$ & $5 \%$ & \\
\hline Ver vídeos para nativos & $20 \%$ & $45 \%$ & $25 \%$ & $5 \%$ & $5 \%$ \\
\hline
\end{tabular}

\section{Conclusiones}

Este artículo es parte de una investigación mixta multifase (Creswell, 2014, Creswell \& Plano Clark, 2011) que explora el uso de preguntas de comprensión audiovisual integradas en el vídeo como subtítulos y sincronizadas con los fragmentos relevantes (v. v. Casañ Núñez, 2015a, 2015b, 2016, 2017) para el aprendizaje de lenguas extranjeras. El trabajo actual tenía el propósito fundamental de comunicar el diseño y la fiabilidad de un cuestionario sobre la comprensión auditiva/audiovisual para estudiantes adultos de ELE que sería empleado en estudios posteriores. Además, una vez comprobada la estabilidad, se analizaron las respuestas de los informantes para conocer algunas de sus creencias y preferencias. Se puede considerar que las indicaciones durante la administración y la redacción de los ítems fueron comprensibles porque los informantes no manifestaron tener dificultades y porque no se hallaron valores perdidos ni anómalos en los análisis de frecuencias de los ítems. Para determinar la fiabilidad se utilizó la técnica del test-retest. Los coeficientes de correlación de Spearman en los ítems ordinales $\left(r_{s}=.522-.879, p<.05\right)$ y el índice de kappa de Cohen en el ítem nominal $(k=.747, p<.001)$ indicaban que el instrumento era fiable. En cuanto a las preferencias y las creencias, se puede destacar que la mayoría consideró que era importante realizar actividades de comprensión auditiva/audiovisual en el aula y que la imagen del vídeo ayudaba a comprender lo que decían los interlocutores. 
Este trabajo puede contribuir al campo de la lingüística aplicada a la enseñanza de lenguas extranjeras de varias formas. En primer lugar, el instrumento tiene aplicación directa en el estudio cuasiexperimental sobre el uso de preguntas de comprensión audiovisual integradas en el vídeo como subtítulos con estudiantes de Español II y, previa adaptación, en investigaciones análogas con otras muestras de la población. En segundo lugar, el artículo puede ayudar a divulgar e ilustrar la técnica del test-retest para estimar la fiabilidad de cuestionarios en el área del ELE. Aunque esta metodología es común en el desarrollo de estos instrumentos (Cohen et al., 2011), no se han encontrado cuestionarios sobre la comprensión/auditiva en ELE que recurrieran a este procedimiento. En cambio, sí se han localizado ejemplos en la enseñanza del inglés como lengua extranjera (por ejemplo, Chang, 2005; Nowrouzi et al., 2014). En tercer lugar, el estudio complementa la literatura de las creencias y preferencias de los estudiantes de lenguas extranjeras sobre el papel del vídeo en la comprensión auditiva/audiovisual (p. ej. Coniam, 2001; Sueyoshi \& Hardison, 2005; Suvorov, 2008, Wagner, 2010). Por último, los ítems pueden ser útiles para investigadores que estén diseñando cuestionarios sobre el mismo tema.

El estudio contó con algunas limitaciones. Para empezar, la decisión de no aplicar el test-retest a los ítems sociodemográficos por su aparente sencillez no se fundamentó en la literatura. Además, los resultados se basan en las respuestas de un número reducido de participantes. Por último, el método test-retest no es perfecto (De Vaus, 2002). Brown (2001) señala que cabe la posibilidad de que los informantes recuerden las respuestas si el intervalo de tiempo entre administraciones es demasiado breve, o de que cambien de opinión si el lapso es excesivamente largo. Cooper y Schindler (2008) advierten de otro inconveniente: los informantes pueden interesarse por el tema y buscar información relacionada antes del retest. Se eligió un intervalo de dos semanas porque así lo sugerían Brown (2001), De Vaus (2002) y Cooper y Schindler (2008) y porque fue el intervalo utilizado en trabajos similares (Bere \& Bjørkelund, 2009; Garmy et al., 2012).

\section{Agradecimientos}

Deseo expresar mi agradecimiento a los estudiantes de Español II de la Facultad de Letras de la Universidade de Coimbra. También quiero manifestar mi gratitud hacia las profesoras que colaboraron en el desarrollo del cuestionario: Ana Gimeno (Universitat Politècnica de València), Virginia González (Universitat de València) y Cláudia Cravo (Universidade de Coimbra). Asimismo, deseo dar las gracias a José da Silva, Pedro Bello y Vítor Cabrita (Universidade de Coimbra), Jesús Jornet (Universitat de València) y Zélia Fernandes (Produtos e Serviços de Estatística) por las sugerencias recibidas para hacer el análisis de fiabilidad test-retest con SPSS. Cualquier error $\mathrm{u}$ omisión es de mi exclusiva 
responsabilidad. Por último, agradezco a los revisores los comentarios y recomendaciones para mejorar el manuscrito.

\section{Referencias}

Altman, D. G. (1991). Practical statistics for medical research. Londres, Reino Unido: Chapman \& Hall.

Bere, E., \& Bjørkelund, L. A. (2009). Test-retest reliability of a new self reported comprehensive questionnaire measuring frequencies of different modes of adolescents commuting to school and their parents commuting to work - the ATN questionnaire. International Journal of Behavioral Nutrition and Physical Activity, 6(1), 1-5. doi:10.1186/1479-5868-6-68

Brown, J. D. (2001). Using surveys in language programs. Cambridge, Reino Unido: Cambridge University Press.

Casañ Núñez, J. C. (2015a). Un marco teórico sobre el uso de preguntas de comprensión audiovisual integradas en el vídeo como subtítulos: un estudio mixto. MarcoELE, 20, 1-45. Recuperado de http://marcoele.com/comprension-audiovisual-ysubtitulos/

Casañ Núñez, J. C. (2015b). Subtitulación de preguntas de comprensión audiovisual: ejemplificación en una secuencia de Ópera prima de Fernando Trueba. Foroele, 11, 45-56. Recuperado de https://ojs.uv.es/index.php/foroele/article/view/7095

Casañ Núñez, J. C. (2016). Actividades de comprensión audiovisual con preguntas integradas en forma de subtítulos: la opinión de catorce estudiantes universitarios de español lengua extranjera. Skopos, 7, 19-38. Recuperado de https://www.uco.es/ucopress/ojs/index.php/skopos/article/view/6896

Casañ Núñez, J. C. (2017). Testing audiovisual comprehension tasks with questions embedded in videos as subtitles: a pilot multimethod study. The EuroCALL Review. The EUROCALL Review, 25(1), 36-60. doi: doi.org/10.4995/eurocall.2017.7062 Recuperado de https://polipapers.upv.es/index.php/eurocall/article/view/7062

Celce-Murcia, M. \& Olshtain, E. (2000). Discourse and context in language teaching: A guide for language teachers. Cambridge, Reino Unido: Cambridge University Press.

Chang, C. Y. K. (2005). Intuitive-analysis style and EFL listening strategies. Annual Review of Education, Communication and Language Sciences, 2, 1-19. Recuperado de http://research.ncl.ac.uk/ARECLS/vol2 documents/Kelly/kelly.htm

Cohen, J. (1988). Statistical power analysis for the behavioral sciencies ( $2^{\mathrm{a}} \mathrm{ed}$.). Hillsdale, Nueva Jersey, Estados Unidos: Lawrence Erlbaum Associates.

Cohen, L., Manion, L., \& Morrison, K. (2011). Research methods in education (7 $7^{\mathrm{a}}$ ed.). Nueva York, Nueva York, Estados Unidos: Routledge.

Coniam, D. (2001). The use of audio or video comprehension as an assessment instrument in the certification of English language teachers: a case study. System, 29(1), 1-14. doi: 10.1016/S0346-251X(00)00057-9

Cooper, D., \& Schindler, P. (2008). Business research methods (10 ed.). Londres, Reino Unido: McGraw-Hill Higher Education.

Creswell, J. W. (2012). Educational research: Planning, conducting, and evaluating quantitative and qualitative research. Londres, Reino Unido: Pearson. 
Creswell, J. W. (2014). Research design: Qualitative, quantitative, and mixed methods approaches $\left(4^{\mathrm{a}}\right.$ ed.). Thousand Oaks, California, Estados Unidos: Sage Publications.

Creswell, J. W., \& Plano Clark, V. L. (2011). Designing and conducting mixed methods research ( $3^{\mathrm{a}}$ ed.). Londres, Reino Unido: Sage Publications.

De Vaus, D. A. (2002). Surveys in social research (5 ed.). Nueva Gales del Sur, Australia: Allen \& Unwin.

Dörnyei, Z. (2003). Questionnaires in second language research: Construction, administration, and processing. Mahwah, Nueva Jersey, Estados Unidos: Lawrence Erlbaum Associates, Publishers.

Dörnyei, Z. (2007). Research methods in applied linguistics. Oxford, Reino Unido: Oxford University Press.

Field, A. (2009). Discovering statistics using SPSS ( $3^{\text {a }}$ ed.). Londres, Reino Unido: Sage publications.

Field, J. (2008). Listening in the language classroom. Cambridge, Reino Unido: Cambridge University Press.

Flowerdew, J., \& Miller, L. (2005). Second language listening. Nueva York, Nueva York, Estados Unidos: Cambridge University Press.

García-Romeu, J. ( 2006). Análisis de necesidades para la programación de cursos de fines específicos. En A. Escofet, B. de Jonge, A. van Hooft, K. Jauregui, J. Robisco, \& M. Ruiz (eds.), Actas del III CIEFE (pp. 145-161). Utrecht, Países Bajos: Ministerio de Educación y Ciencia de España. Recuperado de http://cvc.cervantes.es/ensenanza/biblioteca_ele/ciefe/pdf/03/cvc_ciefe_03 0014.pd $\underline{f}$

Garmy, P., Jakobsson, U., \& Nyberg, P. (2011). Development and psychometric evaluation of a new instrument for measuring sleep length and television and computer habits of Swedish school-age children. The Journal of School Nursing, 28(2), 138-143. doi:10.1177/1059840511420878

Giovannini, A., Martín Peris, E., Rodríguez, M., \& Simón, T. (1996). Profesor en acción 3. Destrezas. Madrid, España: Edelsa.

Hammond, A., Tennant, A., Tyson, S. F., Nordenskiöld, U., Hawkins, R., \& Prior, Y. (2015). The reliability and validity of the English version of the evaluation of daily activity questionnaire for people with rheumatoid arthritis. Rheumatology, 54(9), 1605-1615. doi:10.1093/rheumatology/kev008

Harris, T. (2003). Listening with your eyes: The importance of speech-related gestures in the language classroom. Foreign Language Annals, 36(2), 180-187. doi: 10.1111/j.1944-9720.2003.tb01468.x

Krashen, S. D. \& Terrel, T. D. (1983). The natural approach: Language acquisition in the classroom. Oxford: Pergamon Press.

Larson-Hall, J. (2010). A guide to doing statistics in second language research using SPSS. Nueva York, Nueva York, Estados Unidos: Routledge. doi: $10.4324 / 9780203875964$

Lynch, T. (2009). Teaching second language listening. Oxford, Reino Unido: Oxford University Press.

Lynch, T. (2012). Traditional and modern skills. Introduction. En M. Eisenmann \& T. Summer (eds.), Basic issues in EFL teaching and learning (pp. 69-81). Heidelberg, Alemania: Winter.

Masson, L. F., McNeill, G., Tomany, J. O., Simpson, J. A., Peace, H. S., Wei, L., ... Bolton-Smith, C. (2003). Statistical approaches for assessing the relative validity of 
a food-frequency questionnaire: use of correlation coefficients and the kappa statistic. Public Health Nutrition, 6(03). doi:10.1079/phn2002429

Mayer, R. E. (2009). Multimedia learning ( $2^{\mathrm{a}}$ ed). Nueva York, Nueva York, Estados Unidos: Cambridge University Press.

Minera Reyna, L. E. (2010). El cuestionario MAALE, técnica para recolección de datos sobre las variables afectivas motivación y actitudes en el aprendizaje de una lengua extranjera. RedELE: revista electrónica de didáctica del español lengua extranjera, 19, 1-23. Recuperado de

http://www.mecd.gob.es/dctm/redele/Material-

RedEle/Revista/2010_19/2010_redELE_19_03Minera.pdf?documentId=0901e72b8 $\underline{\text { 0dd2779 }}$

Mendelsohn, D. J. (1994). Learning to listen: A strategy based approach for the second language learner. Carlsbad, California, Estados Unidos: Dominie Press.

Murillo Torrecilla, F. J. (2004). Cuestionarios y escalas de actitudes. Madrid, España: Universidad Autónoma de Madrid. Recuperado de http://www.uam.es/personal_pdi/stmaria/jmurillo/Metodos/Materiales/Apuntes $\% 20$ Cuestionario.pdf

Nowrouzi, S., Tam, S. S., Nimehchisalem, V., \& Zareian, G. (2014). Developing an instrument for Iranian EFL learners' listening comprehension problems and listening strategies. Advances in Language and Literacy Studies 5(3), 63-69. Doi:10.7575/aiac.alls.v.5n.3p.63

Ockey, G. J. (2007). Construct implications of including still image or video in computerbased listening tests. Language Testing, 24(4), 517-537. doi: $10.1177 / 0265532207080771$

Pallant, J. (2011). SPSS survival manual: A step by step guide to data analysis using SPSS ( $4^{\text {a }}$ ed.). Maidenhead, Australia: Open University Press/McGraw-Hill.

Plass, J. L. \& Jones, L. (2005). Multimedia learning in second language acquisition. En R. E. Mayer (ed.), The Cambridge handbook of multimedia learning (pp. 467-488). Cambridge, Reino Unido: Cambridge University Press.

Richards, J. C., \& Burns, A. (2012). Tips for teaching listening. Nueva York, Nueva York, Estados Unidos: Pearson Education.

Riley, P. (1979). Viewing comprehension: "L'oeil écoute". Mélanges CRAPEL, 10, 80-95. Recuperado de http://www.atilf.fr/IMG/pdf/melanges/6riley.pdf

Rost, M. (2016). Teaching and researching listening ( $3^{\mathrm{a}}$ ed.). Nueva York, Nueva York, Estados Unidos: Routledge.

Rubin, J. (1995). An overview to A guide for the teaching of second language listening. En D. J. Mendelsohn \& J. Rubin (eds.), A guide for the teaching of second language listening (pp. 7-11). San Diego, California, Estados Unidos: Dominie Press, Inc.

Saris, W. E., \& Gallhofer, I. N. (eds.). (2014). Design, evaluation, and analysis of questionnaires for survey research ( $2^{\mathrm{a}}$ ed.). doi:10.1002/9781118634646

Sueyoshi, A., \& Hardison, D. M. (2005). The role of gestures and facial cues in second language listening comprehension. Language Learning, 55(4), 661-669. doi: $10.1111 / \mathrm{j} .0023-8333.2005 .00320 . \mathrm{x}$

Suvorov, R. S. (2008). Context visuals in L2 listening tests: the effectiveness of photographs and video vs. audio-only format. Retrospective theses and dissertations (paper 15448). Recuperado de http://lib.dr.iastate.edu/cgi/viewcontent.cgi?article=16447\& context=rtd

Suvorov, R. S. (2015). The use of eye tracking in research on video-based second language (L2) listening assessment: A comparison of context videos and content videos. Language Testing, 32(4), 463-483. doi: 10.1177/0265532214562099 
Sweller, J., Ayres, P., \& Kalyuga, S. (2011). Cognitive load theory. Londres, Reino Unido: Springer

Trindade Natel, T. B. (2002). La proximidad entre el portugués y el español, ¿facilita o dificulta el aprendizaje". En Actas del XIII Congreso Internacional de la Asociación para la Enseñanza del Español como Lengua Extranjera (pp. 825-832). Recuperado de http://cvc.cervantes.es/ensenanza/biblioteca_ele/asele/pdf/13/13_0825.pdf

Underwood, M. (1989). Teaching listening. Londres, Reino Unido: Longman.

Ur, P. (1999). A course in language teaching: Practice and theory. Cambridge, Reino Unido: Cambridge University Press.

Vandergrift, L., \& Goh, C. C. M. (2012). Teaching and learning second language listening. Nueva York, Nueva York, Estados Unidos: Routledge.

Wagner, E. (2007). Are they watching? Test-taker viewing behavior during an L2 video listening test. Language Learning and Technology, 11(1), 67-86. Recuperado de http://lit.msu.edu/vol11num1/pdf/wagner.pdf

Wagner, E. (2010). Test-takers' interaction with an L2 video listening test. System, 38(2), 280-291. doi: 10.1016/j.system.2010.01.003

\section{Anexo}

\section{Cuestionario sobre el aprendizaje de la comprensión auditiva/audiovisual}

\section{Nombre:}

El presente cuestionario tiene por objeto recoger tu opinión sobre algunos aspectos relacionados con el aprendizaje de la comprensión auditiva/audiovisual para mejorar su enseñanza y aprendizaje. Las respuestas son confidenciales y solo se utilizarán con fines académicos. No hay respuestas correctas ni incorrectas porque se basan en tus experiencias particulares como aprendiente de español como lengua extranjera.

\section{Preguntas generales}

1. ¿Cuántos años tienes? Señala la respuesta con un círculo.
1) 24 o menos
2) 25-29 años
3) 30-34 años
4) 35-39 años
5) 40-44 años
6) 45-49 años
7) 50-54 años
8) 55 o más

2. ¿Qué licenciatura estás estudiando? Señala la respuesta con un círculo.
1) Arqueología e Historia
2) Derecho
3) Estudios Artísticos
4) Estudios Clásicos
5) Estudios Europeos
6) Periodismo ("Jornalismo")
7) Portugués
8) Psicología
9) Turismo
10) Otra, ¿cuál?

3. ¿Has estudiado otra licenciatura anteriormente? Señala la respuesta con un círculo.
1) Sí
2) No 
4. En caso afirmativo, ¿qué licenciatura?

5. ¿Cuál es tu lengua materna? Señala la respuesta con un círculo.
1) Portugués
2) Inglés
3) Francés
4) Alemán
5) Italiano

6) Otra, ¿cuál?

6. ¿Has estudiado en la FLUC? Señala la respuesta con un círculo.

1) Sí 2) No

7. ¿Cuánto tiempo has estudiado español a lo largo de tu vida aproximadamente?

8. ¿Has estado en países hispanohablantes (España, México, etc.)? Señala la respuesta con un círculo.
1) Sí
2) No

9. En caso afirmativo, ¿cuánto tiempo aproximadamente?

\section{Preferencias de aprendizaje}

10. ¿Qué importancia tiene para ti practicar la comprensión auditiva/audiovisual en clase para aprender español? Señala la respuesta con un círculo.
1) poquísima
2) poca
3) ni mucha ni poca
4) mucha
5) muchísima

11. Cuando se realiza una actividad de comprensión audiovisual en clase, ¿el tema del vídeo influye positivamente en tu motivación en la actividad? Señala la respuesta con un círculo.
1) poquísima
2) poca
3) ni mucha ni poca
4) mucha
5) muchísima

12-18. Valora de 1 a 5 el interés de los siguientes temas para ti. Completa la tabla poniendo X.
1) poquísima
2) poca
3) ni mucha ni poca
4) mucha
5) muchísima

\begin{tabular}{l|l|l|l|l|l} 
& 1 & 2 & 3 & 4 & 5 \\
\hline 12. Cine & & & & & \\
\hline 13. Cocina & & & & & \\
\hline 14. Deporte & & & & & \\
\hline 15. Historia & & & & & \\
\hline 16. Música & & & & & \\
\hline 17. Noticias de actualidad & & & & & \\
\hline 18. Fiestas tradicionales & & & & &
\end{tabular}

19. Sobre la base de tu experiencia como estudiante de español, ¿qué tipo de grabaciones prefieres para practicar la comprensión auditiva/audiovisual en el aula? Señala con un círculo la opción que prefieras.

1) Prefiero las grabaciones de audio

2) Prefiero las grabaciones de vídeo 
3) Ambas por igual

\section{Valor de la imagen}

20. Según tu experiencia aprendiendo lenguas extranjeras, ¿la imagen del vídeo te ayuda a comprender lo que están diciendo los interlocutores? Señala la respuesta con un círculo.
1) poquísima
2) poca
3) ni mucha ni poca
4) mucha
5) muchísima

\section{Aprendizaje fuera del aula}

21-26. Hay muchas asignaturas ("cadeiras") y hay que repartir el tiempo de estudio entre todas. A continuación hay una lista de actividades para mejorar la comprensión auditiva/audiovisual en español fuera del aula. Señala la frecuencia semanal de 1 a 5. Completa la tabla poniendo X.
1) No lo hago
2) 1 o 2 veces
3) 3 o 4 veces
4) 5 o 6 veces
5) todos los días

\begin{tabular}{|c|c|c|c|c|c|}
\hline & 1 & 2 & 3 & 4 & 5 \\
\hline $\begin{array}{l}\text { 21. Escuchar grabaciones de audio específicas para personas } \\
\text { que aprenden español como lengua extranjera (grabaciones } \\
\text { de manuales) }\end{array}$ & & & & & \\
\hline $\begin{array}{l}\text { 22. Escuchar grabaciones de audio para hablantes nativos de } \\
\text { español (radio de países hispanohablantes, canciones de } \\
\text { grupos de música españoles, etc.) }\end{array}$ & & & & & \\
\hline 23. Hablar con nativos & & & & & \\
\hline 24. Hablar con personas no nativas en español & & & & & \\
\hline $\begin{array}{l}\text { 25. Ver vídeos específicos para personas que aprenden } \\
\text { español como lengua extranjera }\end{array}$ & & & & & \\
\hline $\begin{array}{l}\text { 26. Ver vídeos para hablantes nativos (televisión de España, } \\
\text { películas de países hispanohablantes, etc.) }\end{array}$ & & & & & \\
\hline
\end{tabular}

\section{Información adicional}

A continuación tienes un espacio en el que puedes hacer cualquier comentario (en español o portugués) que consideres oportuno sobre el aprendizaje de la comprensión auditiva/audiovisual.

\footnotetext{
Biodata:

Juan Carlos Casañ Núñez es doctor internacional en Lenguas y Tecnología por la Universitat Politècnica de València. Es máster en enseñanza del español como lengua extranjera (ELE) por la Universidad Internacional Menéndez Pelayo - Instituto Cervantes, así como licenciado en Filología Hispánica por la Universitat de València y en Filología Inglesa por la Universidad Jaume I. Sus intereses de investigación son la enseñanza/aprendizaje de lenguas extranjeras, la comprensión auditiva/audiovisual, la metodología de la investigación, y la sociolingüística. Ha desarrollado su labor profesional en las áreas de la enseñanza del ELE y de la educación en España, Estados Unidos, Francia, Polonia y Portugal.
}

Email: juancarloscasan@protonmail.com

Para citar este artículo:

Casañ Núñez, J.C (2017). Diseño y fiabilidad de un cuestionario sobre la comprensión auditiva/audiovisual. Bellaterra Journal of Teaching \& Learning Language \& Literature, 10(3), 47-65. DOI: http://doi.org/10.5565/rev/jt13.686

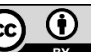

